\section{Arbitrarily Low Sensitivity (ALS) in Linear Distributed Systems Using Pointwise Linear Feedback}

\section{MATEI KELEMEN, YAKAR KANNAI, AND ISAAC HOROWITZ}

Abstract-One of the major reasons for using feedback is to reduce the sensitivity of systems to (uncertain) plant parameters.

In this note, the sensitivity problem is defined for feedback systems with plants described by linear partial differential operators having constant coefficients in a bounded one-dimensional domain. There are also finitely many observation points (and also finitely many lumped feedback loops) and a finite number of (disturbance) inputs.

The sensitivity problem is then studied in detail for the heat equation, and comments are made about the linearized (damped) beam equation and the (damped) wave equation.

It will be shown that it is possible to reduce, arbitrarily, the sensitivity over any (temporal) frequency interval, uniformly in the space domain (except for the undamped wave equation, where a limitation in the frequency interval is induced by the plant).

This reduction may require high-gain feedback around the points where the disturbances appear.

Finally, the negative effect (on ALS) of poor knowledge of the disturbance input points is discussed, and possible solutions are suggested.

\section{Introduction and Formulation of the Problem}

Along with stabilization and disturbance attenuation, the reduction of sensitivity of a system to (uncertain) plant parameters is one of the major reasons for using feedback.

In this note, we approach the sensitivity problem for a plant described by a linear partial differential operator $P$ in the space variable $x \in \mathbb{D}=$ $[0, \pi]$ and the time variable $t>0$. The approach we shall present to sensitivity reduction will be useful, also, in dealing with the disturbance attenuation problem.

We shall assume that the coefficients of $P$ are independent of $t$. Both the input $u$ and the output $y$ of the plant are functions of $x$ and $t$. We assume that the boundary conditions imposed on $y$ are so as to ensure the well-posedness of the problem of finding $y$ when $u$ belongs to a certain class of functions (specified below). For design reasons, we shall work with the Laplace transform (with respect to the $t$ variable). As usual, we denote the transform variable by $s$ and use capital letters for the transformed functions.

Note that our model differs from the one considered in [1]. There, plant described by a fixed irrational matrix was desensitized by (lumped) feedback, whereas, we are interested in reducing sensitivity over all the space range of the plant using point feedback loops properly located.

We assume, now that the transfer function $P_{x_{1} x_{2}}(s)$ is known for al $x_{1}, x_{2} \in \mathbb{D}, P_{x_{1} x_{2}}(s)$ is the transfer function between an input at $x_{1}$ and output at $x_{2}$.

This "plant" is embedded in a feedback structure having $n$ point feedback loops, i.e., each with the sensor and actuator both located at a poin $x_{0_{j}}, j=1, \cdots, n$. We shall consider $0<x_{0_{1}}<x_{0_{2}}<, \cdots,<x_{0_{n}}<\pi$. There is, also, a Laplace transformable disturbance input

$$
u(x, t)=\sum_{j=1}^{m} u_{x_{i_{j}}}(t)\left(\delta_{x=x_{i_{j}}}\right)
$$

where $0<x_{i_{1}}<\cdots<x_{i_{m}}<\pi$. Since we do not want to alter the steady

Manuscript received June 22, 1988; revised July 29, 1989. Paper recommended by Past Associate Editor, T. J. Tarn.

M. Kelemen and Y. Kannai are with the Department of Mathematics. The Weizmann Institute of Science, Rehovot 76100 , Israel

I. Horowitz is with the Department of Mathematics, The Weizmann Institute of Sci ence, Rehovot 76100, Israel, and the Department of Electrical Engineering, University of California, Davis, CA 95616.

IEEE Log Number 9036546. state of the system, we shall assume that all the functions $u_{x_{i_{j}}}(t)$ go to zero as $t$ tends to infinity.

We compute now the transform matrix of the closed-loop $T_{x_{i} x}(s)$ from this input to the outputs at the points $x_{1}, \cdots, x_{r}$ (in D)

$$
\begin{array}{ll}
Y_{x_{k}}(s)=\sum_{j=1}^{m} P_{x_{i_{j}} x_{k}}(s) U_{x_{i j}}(s)+\sum_{j=1}^{n} P_{x_{0_{j}} x_{k}}(s) U_{x_{0_{j}}}(s), & k=1, \cdots, r \\
U_{x_{0_{j}}}(s)=-G_{j}(s) Y_{x_{0_{j}}}(s), & j=1, \cdots, n \\
Y_{x_{0_{j}}}(s)=\sum_{i=1}^{m} P_{x_{i_{i} x_{0_{j}}}}(s) U_{x_{i_{j}}}(s)+\sum_{i=1}^{n} P_{x_{0_{j}} x_{0_{j}}}(s) U_{x_{0_{i}}}(s) . &
\end{array}
$$

Here, the first and the third equations are (Laplace transform) responses at the points $x_{k}$ and $x_{0 j}$, respectively, due to the disturbance and feedback inputs, while the second equation describes the feedback loops, having the compensators $G_{j}(s)$.

In matrix form, we have

$$
\begin{aligned}
Y_{x}(s)= & P_{x_{i} x}(s) U_{x_{i}}(s)+P_{x_{0} x}(s) U_{x_{0}}(s) \\
& U_{x_{0}}(s)=-G(s) Y_{x_{0}}(s) \\
Y_{x_{0}}(s)= & P_{x_{i} x_{0}}(s) U_{x_{i}}(s)+P_{x_{0} x_{0}}(s) U_{x_{0}}(s)
\end{aligned}
$$

where $x=\left(x_{1}, \cdots, x_{r}\right), x_{i}=\left(x_{i_{1}}, \cdots, x_{i_{m}}\right), x_{0}=\left(x_{0_{1}}, \cdots, x_{0_{n}}\right)$

$$
G(s)=\operatorname{diag}\left(G_{1}(s), \cdots, G_{n}(s)\right)
$$$$
Y_{x}(s)=\operatorname{col}\left(Y_{x_{1}}(s), \cdots, Y_{x_{r}}(s)\right)
$$

$$
P_{x_{i} x}(s)=\left[\begin{array}{ccc}
P_{x_{i_{1}} x_{1}}(s) & \cdots & P_{x_{i_{m}} x_{1}}(s) \\
\vdots & & \vdots \\
P_{x_{i_{1}} x_{r}}(s) & \cdots & P_{x_{i_{m}} x_{r}}(s)
\end{array}\right]
$$

and similarly for the other vectors and matrices.

By eliminating $U_{x_{0}}$ and $Y_{x_{0}}$ we obtain

$Y_{x}(s)=\left(P_{x_{i} x}(s)-P_{x_{0} x}(s)(I+L(s))^{-1} L(s) P_{x_{0} x_{0}}^{-1}(s) P_{x_{i} x_{0}}(s)\right) U_{x_{i}}(s)$.

Hence, the transfer matrix is

$$
T_{x_{i} x}(s)=P_{x_{i} x}(s)-P_{x_{0} x}(s)(I+L(s))^{-1} L(s) P_{x_{0} x_{0}}^{-1}(s) P_{x_{i} x_{0}}(s)
$$

where $L(s)=G(s) P_{x_{0} x_{0}}(s)$ is the loop transmission matrix and $I$ is the unit matrix.

The effect of the feedback on the initial plant $P_{x_{i} x}(s)$ is given by

$$
T_{x_{i} x}(s)=P_{x_{i} x}(s) S_{x_{i} x}(s) .
$$

Using (2) and assuming now $m=r$, we obtain

$$
S_{x_{i} x}(s)=I-P_{x_{i} x}^{-1}(s) P_{x_{0} x}(s)(I+L(s))^{-1} L(s) P_{x_{0} x_{0}}^{-1}(s) P_{x_{i} x_{0}}(s)
$$

Definition: We call $S_{x_{i} x}(s)$ the sensitivity function corresponding to $x_{i}$ and $x$.

Remark 1.1: When $x_{i}=x_{0}$ we recover the well-known sensitivity formula (see, for instance, [1])

$$
S=(I+L)^{-1} \text {. }
$$

Another justification of this definition is that if $S_{x_{i} x}(s)$ is kept "small" on a given (temporal) frequency range, then $T_{x_{i} x}(s)$ is less sensitive than $P_{x_{i} x}(s)$ to uncertain parameters, on that range [see (3)].

We now state the problem of achieving arbitrarily low sensitivity (ALS).

ALS Problem: Given $x_{i}, \omega_{0}>0$, and $\epsilon_{0}>0$, under what conditions on $n, x_{0}$ (the number and locations of the loops), and $P$ (the plant), one can find a real rational strictly proper compensator matrix $G(s)$, such that for any $x \in \mathbb{D}^{\prime}$ the following requirements are satisfied.

a) The closed-loop system is stable (i.e., the poles of $T_{x_{i} x}(s)$ are in the left half of the complex plane, bounded away from the imaginary axis, uniformly in $x$ ). (This definition of stability is appropriate for the class of plants and compensators considered here.)

b) $\left|S_{x_{i} x}(i \omega)\right|<\epsilon_{0}$ for $\omega \in\left[-\omega_{0}, \omega_{0}\right]$. 
Here, the vector norm is the Euclidean one and the matrix norm is given by its largest singular value.

In the ODE case, one requires also that for any given $M>1$,

c) $\left|S_{x_{i} x}(i \omega)\right|<M$ for $\omega \in$ S the set of real numbers.

Remark 1.2: It will turn out, in the cases that we shall study, that the requirement $\mathrm{c}$ ) is not satisfied. This is unlike the situation in the (space) continuous feedback (implicit, for instance, in [2]).

\section{Sufficient Conditions for Solving the AlS Problem}

In this section, we shall give a set of conditions on the plant, which will allow the solution of the ALS problem, by properly choosing $x_{0}$ and G.

These conditions are as follows.

i) $P_{x_{i} x}$ is stable, and $P_{x_{0} x_{0}}$ is minimum phase, i.e., there exists a $\sigma_{0}<0$ such that det $P_{x_{0} x_{0}}(s) \neq 0$ for $\operatorname{Re} s \geq \sigma_{0}$.

ii) $P_{y z}(s)$ is Hölder continuous, i.e., $\left|P_{y z}(s)-P_{v z}(s)\right| \leq C|y-v|^{\alpha}$ for some $C>0$ and $0<\alpha<1$; both $\alpha$ and $C$ should be independent of $s(=i \omega)$.

iii) $P_{x_{0} x_{0}}(s)$ is ultimately diagonal: there is a diagonal matrix $D(s)$ such that

$$
\lim _{\bar{R} \rightarrow \infty} \sup _{\substack{|s| \geq \bar{R} \\ \operatorname{Re} s \geq 0}}\left|\left(P_{x_{0} x_{0}}(s)-D(s)\right) D^{-1}(s)\right|=0 .
$$

iv) $\operatorname{det} P_{x_{i} x}(i \omega) \neq 0$ for $\omega \in$ 's.

We claim that the ALS problem can be solved, i.e., that the properties a) and b) from Section I hold, if the conditions i)-iv) are satisfied.

Indeed, the property a) (stability) can be satisfied in view of the condition i). Moreover, due to [1, Theorem 1] (which, roughly speaking, states that one can use "high gain" feedback without damaging stability in a certain class of systems described by minimum-phase transfer matrices), the stability of the closed-loop is preserved even when using an arbitrarily high gain (properly chosen) compensator $G$. To apply this theorem, we also need the condition iii).

As for the property b) (of ALS), we shall use the following inequality, derived from (4) (we shall omit the argument $i \omega$ )

$$
\begin{aligned}
\left|S_{x_{i} x}\right| \leq & \left|P_{x_{i} x}^{-1}\right|\left|P_{x_{0} x}-P_{x_{i} x}\right|+\left|P_{x_{0} x_{0}}^{-1}\right|\left|P_{x_{i} x_{0}}-P_{x_{i} x_{0}}\right|+\left|(I+L)^{-1}\right| \\
& +\left|(I+L)^{-1}\right|\left|P_{x_{0} x_{0}}^{-1}\right|\left|P_{x_{i} x_{0}}-P_{x_{0} x_{0}}\right| \\
& +\left|P_{x_{i} x}^{-1}\right|\left|P_{x_{0} x}-P_{x_{i} x}\right|\left|(I+L)^{-1}\right| \\
& +\left|P_{x_{i} x}^{-1}\right|\left|P_{x_{0} x}-P_{x_{i} x}\right|\left|P_{x_{u} x_{0}}^{-1}\right|\left|P_{x_{i} x_{0}}-P_{x_{0} x_{0}}\right| \\
& +\left|P_{x_{i} x}^{-1}\right|\left|P_{x_{0} x}-P_{x_{i} x}\right|\left|(I+L)^{-1}\right|\left|P_{x_{i}, x_{0}}^{-1}\right|\left|P_{x_{i} x_{0}}-P_{x_{0} x_{0}}\right| .
\end{aligned}
$$

Of course, we assumed here that $m=r=n$.

Now i) and iv) assure the boundedness of $\left|P_{x_{i} x}^{-1}\right|$ and $\left|P_{x_{0} x_{0}}^{-1}\right|$ on the compact set $\left[-\omega_{0}, \omega_{0}\right]$. Together with ii), the terms in $\left(4^{\prime}\right)$ which do not contain the factor $\left|(I+L)^{-1}\right|$ can be made arbitrarily small, if $\left|x_{0}-x_{i}\right|$ is small enough.

Again, with [1, Theorem 1] (which is applicable if i) and iii) are satisfied), the terms in $\left(4^{\prime}\right)$ containing the factor $\left|\left(I+L^{-1}\right)\right|$ can be made arbitrarily small too, with a high-gain type $G(s)$. Thus, the requirement b) (achieving ALS on $\left.\left[-\omega_{0}, \omega_{0}\right]\right)$ is also satisfied.

Remark 2.1: It follows from (2), that by point feedback, we cannot move the poles of the plant, hence, we cannot stabilize an unstable system.

Remark 2.2: The fact that $\alpha$ and $C$ from condition ii) are independent of $s$, will assure that the number and position of the feedback loops ( $n$ and $x_{0}$, respectively), and the compensator $G$ which may solve the ALS problem, are independent of $\omega_{0}$.

Remark 2.3: Requirement c) can be satisfied when $x_{i}=x_{0}$, for instance. However, we shall show by a counterexample (for the heat equation), that $c$ ) is not generally valid.

But even if c) were not true, it follows from (2) that

$$
\lim _{\substack{s \rightarrow \infty \\ \operatorname{Re} s \geq 0}} T_{x_{i} x}(s)=P_{x_{i} x}(s)
$$

uniformly in $x$ and $x_{i}$ (if $P_{x_{i} x}$ is stable and proper). Hence, at high frequencies, the closed-loop behavior is not worse than that of the openloop (just as in the ODE case).

Moreover, the fact that $S_{x, x}(s)$ may tend to infinity (when $s$ goes to infinity), comes from the factor $P_{x_{i}}^{-1}(s)$ and not from $(I+L(s))^{-1}$. So, the stability margin of the system is not endangered, unlike in the ODE case.

\section{EXAMPLes}

\section{A. Heat, (Damped) Wave, and (Damped) Beam Equations}

In this section, we shall check to what extent the conditions i)-iv) from the previous section are satisfied by the plants mentioned above.

Let us begin with the heat equation. The equation of the plant is

$$
\begin{gathered}
\frac{\partial y}{\partial t}-\frac{\partial^{2} y}{\partial x^{2}}=u(x, t), x \in(0, \pi), \quad t>0 \\
y(x, 0)=0, x \in[0, \pi] \\
y(0, t)=y(\pi, t)=0,
\end{gathered}
$$

where $u(x, t)$ is as in (1).

We denote by $P_{y_{j} z_{i}}(s)$ the general element of the matrices we will work with. It is convenient to use it in two different forms (see [3]),

and

$$
p_{y, z_{i}}(s)=4 \sum_{k=1}^{\infty} \frac{1}{s+k^{2}} \sin k y_{j} \sin k z_{l}
$$

$$
P_{y, z_{l}}(s)=\frac{1}{\sqrt{s}} \frac{\left(e^{\sqrt{s\left(\pi-y_{j}\right)}}-e^{-\sqrt{s}\left(\pi-y_{j}\right)}\right)\left(e^{\sqrt{s} z_{i}}-e^{-\sqrt{s} z_{l}}\right)}{2\left(e^{\sqrt{s} \pi}-e^{-\sqrt{s} \pi}\right)}
$$

if $0 \leq z_{l} \leq y_{j}$; for $y_{j} \leq z_{l} \leq \pi$ the transfer function is also given by $\left(5^{\prime}\right)$ but with $z_{l}$ changed in $\pi-z_{l}$ and $\pi-y_{j}$ in $y_{j}$. Then, we have the following.

Lemma: The conditions i)-iv) are satisfied for the heat equation plant. We start by checking condition i). Using (5) we can write, if $s=\sigma+i \omega$, that

$$
\begin{aligned}
& P_{x_{0} x_{0}}(s)=4\left[\begin{array}{cccc}
\sum_{k=1}^{\infty} \frac{\sigma+k^{2}}{\left|s+k^{2}\right|^{2}} \sin k x_{0_{1}} & \sin k x_{0_{1}} & \cdots & \sum_{k=1}^{\infty} \frac{\sigma+k^{2}}{\left|s+k^{2}\right|^{2}} \sin k x_{0_{n}} \sin k x_{0_{1}} \\
\vdots & \vdots \\
\sum_{k=1}^{\infty} \frac{\sigma+k^{2}}{\left|s+k^{2}\right|^{2}} \sin k x_{0_{1}} & \sin k x_{0_{n}} & \cdots & \sum_{k=1}^{\infty} \frac{\sigma+k^{2}}{\left|s+k^{2}\right|^{2}} \sin k x_{0_{n}} \sin k x_{0_{n}}
\end{array}\right] \\
& +4 i \omega\left[\begin{array}{cccc}
\sum_{k=1}^{\infty} \frac{1}{\left|s+k^{2}\right|^{2}} \sin k x_{0_{1}} \sin k x_{0_{1}} & \cdots & \sum_{k=1}^{\infty} \frac{1}{\left|s+k^{2}\right|^{2}} \sin k x_{0_{n}} \sin k x_{0_{1}} \\
\vdots & \vdots \\
\sum_{k=1}^{\infty} \frac{1}{\left|s+k^{2}\right|^{2}} \sin k x_{0_{1}} \sin k x_{0_{n}} & \cdots & \sum_{k=1}^{\infty} \frac{1}{\left|s+k^{2}\right|^{2}} \sin k x_{0_{n}} \sin k x_{0_{n}}
\end{array}\right] .
\end{aligned}
$$


We shall use exercise 1 in Section 5, Chapter 8 of [4] to prove that $\operatorname{det} P_{x_{0} x_{0}}(s) \neq 0$ for $\sigma>-1$.

Indeed, the imaginary part is real and symmetric. To prove that the real part is positive definite, it is enough to show that all the determinants of $D_{q}$ (the matrix obtained from the initial one by deleting the last $n-q$ rows and columns) $q=1, \cdots, n$ are positive.

Now, if we consider the $l_{2}$ vectors

$$
\begin{aligned}
& V_{1}=\left(\frac{\sqrt{\sigma+1}}{|s+1|} \sin x_{0_{1}}, \cdots, \frac{\sqrt{\sigma+k^{2}}}{\left|s+k^{2}\right|} \sin k x_{0_{0}}, \cdots\right) \\
& \vdots \\
& V_{q}=\left(\frac{\sqrt{\sigma+1}}{|s+1|} \sin x_{0_{q}}, \cdots, \frac{\sqrt{\sigma+k^{2}}}{\left|s+k^{2}\right|} \sin k x_{0_{q}}, \cdots\right)
\end{aligned}
$$

then

$$
D_{q}=\left[\begin{array}{ccc}
\left\langle V_{1}, V_{1}\right\rangle & \cdots & \left\langle V_{1}, V_{q}\right\rangle \\
\vdots & & \vdots \\
\left\langle V_{1}, V_{q}\right\rangle & \cdots & \left\langle V_{q}, V_{q}\right\rangle
\end{array}\right] .
$$

With another result of [4], in Chapter 4 , Section 4 , it follows that all det $D_{q}>0$, if $V_{1}, \cdots, V_{q}$ are linearly independent.

But this is true if the determinants of all

$$
D_{q}^{\prime}=\left[\begin{array}{ccc}
\sin x_{0_{1}} & \cdots & \sin q x_{0_{1}} \\
\vdots & & \vdots \\
\sin x_{0_{q}} & \cdots & \sin q x_{0_{q}}
\end{array}\right]
$$

are nonzero.

By induction we obtain that

$$
\begin{aligned}
& \operatorname{det} D_{q}^{\prime}=2^{q-1} 2^{q-2} \cdots 2 \sin x_{0_{1}} \cdots \sin x_{0_{q}} \\
& \cdot \operatorname{det}\left[\begin{array}{ccc}
1 & \cdots & 1 \\
\cos x_{0_{1}} & \cdots & \cos x_{0_{q}} \\
\cos ^{2} x_{0_{1}} & \cdots & \cos ^{2} x_{0_{q}} \\
\vdots & & \vdots \\
\cos ^{q-1} x_{0_{1}} & \cdots & \cos ^{q-1} x_{0_{q}}
\end{array}\right]
\end{aligned}
$$

which is nonzero since $0<x_{0_{1}}, \cdots, x_{0_{g}}<\pi$, since they are distinct and the determinant on the right-hand side is Vandermonde.

We now check condition ii). According to (5) (here, $y_{1}, y_{2}, z$ are scalars and $\operatorname{Re} s>0$ )

$$
\begin{aligned}
\left|P_{y_{1} z}(s)-P_{y_{2} z}(s)\right| & \leq 8 \sum_{k=1}^{\infty} \frac{1}{k^{2}}\left|\sin \frac{k}{2}\left(y_{1}-y_{2}\right)\right| \\
& \leq 8 \sum_{k=1}^{\infty} \frac{1}{k^{2}}\left(\frac{k}{2}\right)^{1-\beta}\left|y_{1}-y_{2}\right|^{1-\beta}=C_{1}\left|y_{1}-y_{2}\right|^{1-\beta}
\end{aligned}
$$

since for $\beta \in(0,1)$

$$
|\sin v| \leq|v|^{1-\beta}
$$

(Indeed, raise the above inequality to the power $1 / 1-\beta$.) We also used the following inequality, valid for $\operatorname{Re} s \geq 0$

$$
\left|\frac{1}{s+k^{2}}\right| \leq \frac{1}{k^{2}} .
$$

Then ii) is true, since the matrix norm we used is dominated by $n$ times the maximum magnitude entry.

To check condition iii), we shall use $\left(5^{\prime}\right)$. As matrix $D$, we shall take
$P_{X_{0} X_{i}}(s)$ with the off-diagonal elements made zero. Now, if $i<j$

$$
\begin{aligned}
& \lim _{\bar{R} \rightarrow \infty} \sup _{\substack{|s| \geq \bar{R} \\
\operatorname{Re} s \geq 0}}\left|\frac{P_{x_{0_{j}} x_{0_{i}}}(s)}{P_{x_{0_{i}} x_{0_{i}}}(s)}\right| \\
& =\lim _{\bar{R} \rightarrow \infty} \sup _{\substack{|s| \geq \bar{R} \\
\operatorname{Re} s \geq 0}}\left|e^{\sqrt{s\left(x_{0}-x_{0}\right)}}\right|=0
\end{aligned}
$$

since $x_{0_{i}}<x_{0_{j}}$ for $i<j$. (It was enough to consider $i<j$ because $P_{x_{0} x_{0}}$ is symmetric.) Thus, iii) is true.

To check condition iv), we take advantage of the fact that in this particular case, we are able to compute explicitly the zeros of $\operatorname{det} P_{x_{i} x}(s)$.

Let us denote

$$
x_{i_{0}}=0, \quad x_{i_{m+1}}=\pi .
$$

Then, we shall prove (a bit later) that if $s \neq 0$

$$
\begin{aligned}
\operatorname{det} P_{x_{i} x}(s)= & (-1)^{m+1}\left(2 \sqrt{s}\left(e^{\sqrt{s} \pi}-e^{-\sqrt{s} \pi}\right)\right)^{-m}\left(e^{\sqrt{s} x_{1}}-e^{-\sqrt{s} x_{1}}\right) \\
& \times\left(e^{\sqrt{s}\left(\pi-x_{i m}\right)}-e^{-\sqrt{s}\left(\pi-x_{i m}\right)}\right) \\
& \times \prod_{k=2}^{m}\left(e^{\sqrt{s} a_{k}}+e^{-\sqrt{s} a_{k}}-e^{\sqrt{s} b_{k}}-e^{-\sqrt{s} b_{k}}\right)
\end{aligned}
$$

for

$$
\begin{gathered}
x_{i_{k-1}} \leq x_{k} \leq x_{i_{k}} \\
a_{k}=\pi-x_{k}+x_{i_{k-1}} \\
b_{k}=\pi+x_{k}-x_{i_{k-1}}
\end{gathered}
$$

and $k=1, \cdots, m$.

Similarly, for

$$
x_{i_{k}} \leq x_{k}^{\prime} \leq x_{i_{k-1}}
$$

and $k=1, \cdots, m$, then, if $s \neq 0$

$$
\begin{aligned}
\operatorname{det} P_{x_{i} x^{\prime}}(s)= & (-1)^{m+1}\left(2 \sqrt{s}\left(e^{\sqrt{s} \pi}-e^{-\sqrt{s} \pi}\right)\right)^{-m} \\
& \times\left(e^{\sqrt{s} x_{i_{1}}}-e^{-\sqrt{s} x_{i_{1}}}\right)\left(e^{\left.\sqrt{s(\pi}-x_{m i}^{\prime}\right)}-e^{\sqrt{s}\left(\pi-x_{m}^{\prime}\right)}\right) \\
& \times \prod_{k=2}^{m}\left(e^{\sqrt{s} a_{k}^{\prime}}+e^{-\sqrt{s} a_{k}^{\prime}}-e^{\sqrt{s} b_{k}^{\prime}}-e^{-\sqrt{s} b_{k}^{\prime}}\right)
\end{aligned}
$$

where

$$
\begin{aligned}
& a_{k}^{\prime}=\pi-x_{i_{k+1}}+x_{k}^{\prime} \\
& b_{k}^{\prime}=\pi+x_{i_{k+1}}-x_{k}^{\prime} .
\end{aligned}
$$

Hence,

Proposition: det $P_{x_{i} x}(s)$ has zeros on the imaginary axis if and only if at least one of the equalities

$$
x_{k}=x_{i_{k}-1}
$$

$k=1, \cdots, m$ is satisfied. Also, $\operatorname{det} P_{x_{i} x^{\prime}}(s)$ has zeros on the imaginary axis if and only if at least one of the equalities

$$
x_{k}^{\prime}=x_{i_{k+1}}
$$

$k=1, \cdots, m$ is satisfied

Indeed, this is obtained by solving algebraic equations of order two in $e^{-\sqrt{s} a_{k}}$ for $(6)$, and in $e^{-\sqrt{s} a_{k}^{\prime}}$ for $\left(6^{\prime}\right)$. When $s=0$, the same result is true by l'Hôpital's rule.

Note, that when condition $(8)\left[\left(8^{\prime}\right)\right]$ is met, then $\operatorname{det} P_{x_{i} x}(s)$ (det $P_{x_{i} x^{\prime}}(s)$ ) is, in fact, identically zero, on the whole complex plane c.

We are left now with the proof of formula (6). (The proof of $\left(6^{\prime}\right)$ is similar.) 
Let us define

$$
\begin{gathered}
c_{j}=e^{\sqrt{s\left(x-x_{i_{j}}\right)}-e^{-\sqrt{s}\left(x-x_{i j}\right)}} \\
\tilde{c}_{j}=e^{\sqrt{s x_{i_{j}}}}-e^{-\sqrt{s} x_{i_{i}}} \\
d_{j}=e^{\sqrt{s\left(x-x_{j}\right)}-e^{-\sqrt{s}\left(x-x_{j}\right)}} \\
\tilde{d}_{j}=e^{\sqrt{s x_{j}}}-e^{-\sqrt{s} x_{j}}
\end{gathered}
$$

$j=1, \cdots, m$

Then using (7) and (5') we can write

$$
P_{x_{i}} x(s)=\left(2 \sqrt{s}\left(e^{\sqrt{s} \pi}-e^{-\sqrt{s} \pi}\right)\right)^{-m}
$$

$$
\left[\begin{array}{ccccc}
c_{1} \tilde{d}_{1} & c_{2} \tilde{d}_{1} & c_{3} \tilde{d}_{1} & \cdots & c_{m} \tilde{d}_{1} \\
\tilde{c}_{1} d_{2} & c_{2} \tilde{d}_{2} & c_{3} \tilde{d}_{2} & \cdots & c_{m} \tilde{d}_{2} \\
\tilde{c}_{1} d_{3} & \tilde{c}_{2} d_{3} & c_{3} \tilde{d}_{3} & \cdots & c_{m} \tilde{d}_{3} \\
\vdots & & & & \vdots \\
\tilde{c}_{1} d_{m} & \tilde{c}_{2} d_{m} & \tilde{c}_{3} d_{m} & \cdots & c_{m} \tilde{d}_{m}
\end{array}\right]
$$

After factoring out $c_{m} \tilde{d}_{1}$, one subtracts from each row $l=2, \cdots, m$, the first row multiplied by $\tilde{d}_{l}$. The result is a matrix having the last column $(1,0, \cdots, 0)$, and the lower-left $(m-1) \times(m-1)$ submatrix triangular. The diagonal elements of this submatrix are

$$
\tilde{c}_{l-1} d_{l}-c_{l-1} \tilde{d}_{l}
$$

$l=2, \cdots, m$. This finishes the proof of the proposition and of the lemma. Now, from the lemma, the following holds.

Requirement a): Stability, is satisfied. Indeed, the heat equation plant is stable [see (5)], and i) holds.

Requirement $b$ ): Arbitrarily low sensitivity on any given frequency range is satisfied if $\left|x_{0}-x_{i}\right|$ is small enough, and if $x=\left(x_{1}, \cdots, x_{m+1}\right)$ is such

$$
x_{k} \in \text { a compact } \operatorname{set} \subset\left(x_{i_{k-1}}, x_{i_{k}}\right)
$$

$k=1, \cdots, m+1$

Indeed, this follows from i), ii), iii), and from conditions on the nonsingularity of $\boldsymbol{P}_{\boldsymbol{x}_{i} \boldsymbol{x}}$ in iv). (Both cases ( 7$)$ and $\left(7^{\prime}\right)$ have to be considered.)

Observe that if the coefficients of the plant are uncertain, but the conditions i)-iv) from Section II are satisfied uniformly (in the uncertain parameters), then one can design a compensator solving the ALS problem over all the uncertainty range.

Remark 3.1: When one of the conditions $(8)$ or $\left(8^{\prime}\right)$ is met, then the sensitivity function is not even defined because $\operatorname{det} P_{x_{i} x}(s) \equiv 0$ on C [see also (3)]. This is a clear departure from the requirement that $x$ should cover all $\mathbb{D}^{r}$.

Note that the compact set in (9) can be chosen arbitrarily close to the right-hand side set, without having to modify the compensator $G(s)$ [recall formula (4)]. But the larger this compact set, the smaller $\left|x_{i}-x_{0}\right|$ has to be.

Remark 3.2: If one is satisfied with only $x=\left(x_{1}, \cdots, x_{m}\right)$ or $x^{\prime}=$ $\left(x_{2}^{\prime}, \cdots, x_{m+1}^{\prime}\right)$, then even for $x=x_{i}$ (the points where the disturbances occur), ALS is achievable.

Remark 3.3: A significant property is that one can obtain ALS with no more than $m$ (number of inputs) feedback loops. This follows from the particular structure of $\operatorname{det} P_{x_{i} x}(s)$ for the heat equation [see iv)], which aside from conditions $(8)$ and $\left(8^{\prime}\right)$, has no finite zeros.

Remark 3.4: If there is uncertainty also in $x_{i}$, then the (high) price to pay for achieving ALS is to increase the number of loops to assure a small enough $\left|x_{i}-x_{0}\right|$. The bigger the uncertainty, the bigger the number of loops which should be employed. (Then, to keep all the matrices involved square, we have to increase the number of inputs in (1), with a suitable amount of "dummy" (i.e., zero) disturbances.)

Another possibility would be to consider feedback loops "searching" the $x_{i}$ and moving towards it. Moving feedback was also advocated in [5].

We shall end the study of the heat equation by a counterexample to the requirement $\mathrm{c}$ ).
With $G_{1}(s)=\cdots=G_{n}(s)=G(s)$ and $s$ "big," we get from (4)

$$
\left|S_{x_{i} x}(s)-I\right| \approx|G(s)|\left|P_{x_{i} x}^{-1}(s) P_{x_{0} x}(s) P_{x_{i} x_{0}}(s)\right|
$$

since $\left|(I+L(s))^{-1}\right| \approx 1$.

Let $n=m=r=2$ and

$$
0<x_{1}<x_{i_{1}}<x_{0_{1}}<x_{2}<x_{i_{2}}<x_{0_{2}}<\pi .
$$

Then we shall show that for $x_{2}$, in some interval, the right-hand side of the above formula will tend to infinity, when $s$ will go to infinity.

Indeed, for "big" $s$ we have (approximately)

$$
P_{x_{i} x}(s)=\frac{1}{2 \sqrt{s}}\left[\begin{array}{cc}
e^{\sqrt{s}\left(-x_{i_{1}}+x_{1}\right)} & 0 \\
e^{\sqrt{s}\left(-x_{2}+x_{i_{1}}\right)} & e^{\sqrt{s\left(-x_{i_{2}}+x_{2}\right)}}
\end{array}\right]
$$

hence,

$$
P_{x_{i} x}^{-1}(s)=\frac{2 \sqrt{s}}{e^{\sqrt{s\left(x_{1}+x_{2}-x_{i_{1}}-x_{i_{2}}\right)}}}\left[\begin{array}{cc}
e^{\sqrt{s}\left(-x_{i_{2}}+x_{2}\right)} & 0 \\
-e^{\sqrt{s\left(-x_{2}+x_{i_{1}}\right)}} & e^{\sqrt{s\left(-x_{i_{1}}+x_{1}\right)}}
\end{array}\right] ;
$$

also

$$
P_{x_{0} x}(s)=\frac{1}{2 \sqrt{s}}\left[\begin{array}{cc}
e^{\sqrt{s}\left(-x_{\mathrm{x}_{1}}+x_{1}\right)} & 0 \\
e^{\sqrt{s\left(-x_{2}+x_{\hat{v}_{1}}\right)}} & e^{\sqrt{s\left(-x_{0_{2}}+x_{2}\right)}}
\end{array}\right]
$$

and

$$
P_{x_{i} x_{0}}(s)=\frac{1}{2 \sqrt{s}}\left[\begin{array}{cc}
e^{\sqrt{s}\left(-x_{0}+x_{i_{1}}\right)} & e^{\sqrt{s\left(-x_{i_{2}}+x_{0_{1}}\right)}} \\
0 & e^{\sqrt{s\left(-x_{0_{2}}+x_{i_{2}}\right)}}
\end{array}\right] \text {. }
$$

Now, the entry (2), (1) of $P_{x_{i} x}^{-1}(s) P_{x_{0} x}(s) P_{x_{i} x}(s)$ is

$$
\frac{1}{2 \sqrt{s}} e^{\left.\sqrt{s i}-2 x_{2}+x_{i_{1}}+x_{i_{2}}\right)}\left(-e^{2 \sqrt{s}\left(x_{i_{1}}-x_{0_{1}}\right)}+1\right)
$$

which tends to infinity when $s$ goes to infinity, if

$$
x_{0_{1}}<x_{2}<\frac{x_{i_{1}}+x_{i_{2}}}{2} .
$$

This completes the counterexample, since the norm of the matrix is greater than any of its entries.

Next, we consider briefly the wave equation and the beam equation, pointing out the differences from the heat equation.

The equation of the (damped) wave equation plant is

$$
\begin{aligned}
\frac{\partial^{2} y}{\partial t^{2}}-\frac{\partial^{2} y}{\partial x^{2}}+2 \epsilon \frac{\partial y}{\partial t} & =u(x, t), x \in(0, \pi), t>0 \\
y(x, 0) & =0, x \in[0, \pi] \\
\frac{\partial y}{\partial t}(x, 0) & =0, x \in[0, \pi] \\
y(0, t) & =y(\pi, t)=0
\end{aligned}
$$

where $u(x, t)$ is as in (1), and $0 \leq \epsilon<1$ is the damping factor.

One can see that formulas $(5),\left(5^{\prime}\right),(6)$, and $\left(6^{\prime}\right)$ hold even in this case, if $s$ is replaced by $s^{2}+2 \epsilon s$.

We shall denote by $\left(5_{w}\right),\left(5_{w}^{\prime}\right),\left(6_{w}\right)$, and $\left(6_{w}^{\prime}\right)$ the above formulas where $s$ was replaced by $s^{2}+2 \epsilon s(\operatorname{Re} s \geq 0)$.

So, proceeding as in the heat equation case [except for condition ii)], we obtain that all the conclusions and remarks from the heat equation are still valid (i.e., the ALS problem is solvable, and even in an economical way, concerning the number of feedback loops).

Remark 3.5: Instead of condition ii), we proved a weaker one, which still guarantees that ALS is achievable. Namely, taking into account that $P_{x_{i} x}(s)$ has a uniform bounded limit when $s$ tends to infinity (in the first quadrant), we were able to prove that it is, also, uniformly continuous on $[0, \pi]^{2} \times\{s \mid \operatorname{Re} s>0\}$.

Remark 3.6: When $\epsilon=0$, i.e., in the wave equation case, the ALS problem has no solution, regardless of how many feedback loops are used and where they are located. 
Indeed, the Requirement a) (stability) is violated because all the poles of the plant are on the imaginary axis [see $\left(5_{w^{\prime}}\right)$ or $\left.\left(5_{w}^{\prime}\right)\right]$.

Also, Requirement b) (ALS on given frequency range) of the problem is not satisfied for arbitrary $\omega_{0}$, since the properties i) and iv) are not valid. [ det $P_{x_{i} x}(s)$ has zeros on the imaginary axis no matter where $x_{i}$ and $x$ are placed, see $\left(6_{w}\right)$ and $\left(6_{w}^{\prime}\right)$.]

Finally, the equation of the (damped) linearized beam equation plant is

$$
\begin{aligned}
\frac{\partial^{2} y}{\partial t^{2}}-2 \epsilon \frac{\partial^{2} y}{\partial t \partial x^{2}}+\frac{\partial^{4} y}{\partial x^{4}} & =u(x, t), x \in(0, \pi), t>0 \\
y(x, 0) & =0, x \in[0, \pi] \\
\frac{\partial y}{\partial t}(x, 0) & =0, x \in[0, \pi] \\
y(0, t) & =y(\pi, t)=0 \\
\frac{\partial^{2} y}{\partial x^{2}}(0, t) & =\frac{\partial^{2} y}{\partial x^{2}}(\pi, t)=0
\end{aligned}
$$

where $u(x, t)$ is as in (1) and $0 \leq \epsilon<1$ is the damping factor.

As in the heat equation case, we were able to prove that the conditions i)-iii) are satisfied. But unlike the heat equation case, we have no such direct tool for checking condition iv) [i.e., the behavior of $\operatorname{det} P_{x_{i} x}(i \omega)$ ], as formula (6) or $\left(6^{\prime}\right)$. Moreover, we have found that $\operatorname{det} P_{x_{i} x}(s)$ has zeros in the right half plane, for $s$ real and "big." So, it might be possible to detect (experimentally) that $\operatorname{det} P_{x_{i} x}(i \omega)$ has a zero, or becomes undesirably small at some frequency $\omega \in\left[-\omega_{0}, \omega_{0}\right]$. If this is, indeed, the case, then (9) will be no more true for arbitrary compact sets, unless $\left|x_{0}-x_{i}\right|$ is zero, or very small (for the second situation).

A possible way out is to increase the number of inputs (with dummy ones) until $\left|P_{x_{i} x}(i \omega)\right|^{-1}$ is not too big on $\left[-\omega_{0}, \omega_{0}\right]$. This is possible since for $x$ close enough to $x_{i}, P_{x_{i} x}(i \omega)$ is close to $P_{x_{i} x_{i}}(i \omega)$ (on $\left[-\omega_{0}, \omega_{0}\right]$ ), which is known to have nonzero determinant [property i)].

This will call, however, for a corresponding increase in the number of feedback loops (to keep the matrices square).

Thus, ALS is achievable for the damped beam equation, but with (possibly) an increased number of feedback loops. In the undamped case, ALS is no more achievable (see also the undamped wave equation).

\section{Concluding Remarks}

1) We have shown that unlike stability, the sensitivity function of a distributed system can be improved by means of pointwise feedback. In fact, ALS was achievable in the case we studied, the heat equation, the damped beam, and the damped wave equation, but not for the undamped ones.

2) To achieve ALS one has to use high-gain feedback around the points where the disturbances appeared.

3) In some cases, an additional number of feedback loops might be necessary, to compensate for the big values of $\left|P_{x_{i} x}(i \omega)\right|^{-1}$ in $\left(4^{\prime}\right)$. We believe, however, that for achieving ALS, there is no need for much more feedback loops than the number of disturbance inputs existing in the system.

4) If the vector $x_{i}$ (of the points where the disturbances occur) is not precisely known, then ALS is not achievable, i.e., the uncertainty in the parameters of the system will be reduced only by luck.

Possible remedies are an increase in the number of feedback loops around the possible points where disturbances may occur, or mobile feedback loops, "searching" for $x_{i}$.

\section{ACKNOWLEDGMENT}

The authors wish to thank Prof. C. Foias for suggesting the study of the damped wave equation.

REFERENCES

[1] G. Zames and D. Bensoussan, "Multivariable feedback, sensitivity, and decentralized control," IEEE Trans. Automat. Contr., vol. 28, pp. 1030-1035, 1983.
[2] M. Kelemen, Y Kannai, and I. Horowitz, "Improved method for designing linear distributed feedback systems," submitted for publication.

[3] - "One point feedback approach to distributed linear systems," Int. J. Contr., vol. 49, pp. 969-980, 1989.

[4] R. Bellman, Introduction to Matrix Analysis. New York: McGraw-Hill, 1960

[5] A. Bamberger, J. Jaffre, and J. P. Yvon, "Punctual control of vibrating string: Numerical analysis," Comp. Math. Appl., vol. 4, pp. 113-138, 1978.

\section{Optimal Observers for Systems with Colored Noises}

\section{YORAM HALEVI}

Abstract-The problem of optimal full-order observers for continuous-time linear systems with colored process and measurement noises is considered. Optimal estimation of the state, in such cases, involves augmentation of the system, thus, a higher order observer is required. In this note, the structure of a full-order observer is assumed and necessary conditions for the optimal observer are derived. The conditions are given for the general case where the intensity of the white noise component of the measurement noise may be singular. The solution consists of a modified Riccati equation and a Lyapunov equation coupled by two projection matrices in the singular case and one projection matrix in the nonsingular case. The problem under consideration is distinct from that of the optimal reduced-order estimator since, in general, the latter is not an observer.

\section{INTRODUCTION}

The process of optimal state estimation in the presence of colored process and measurement noises consists of two stages. First, the state is augmented to include the states of the shaping filters that generate the colored noises. The resulting augmented system has white process and measurement noises. Then, standard techniques such as the Kalman filter, in case the measurement noise covariance matrix is nonsingular, or singular estimation methods [4], [5], [7], [8], [13] otherwise, are used to obtain the optimal estimate of the augmented state. Hence, the optimal estimator is an observer with dimension higher than that of the original system. While this procedure yields the optimal estimation, the use of higher order observers is undesirable from a practical point of view. In many cases, the states of the shaping filters are merely a mathematical fiction and, as such, their estimates, which necessarily accompany the estimate of the original state, are of no interest. Furthermore, if the spectra of the noises are obtained experimentally, then better approximations require higher orders of the shaping filters, which, in turn, implies a further increase in the dimension of the observer.

A different approach to the problem is considered in this note. The structure of a full-order observer, having the same dimension as the original system, is assumed and the optimal gain which minimizes a quadratic error criterion is sought. This approach enables the use of accurate high-order stochastic models of the colored noises without adding to the complexity of the resulting observer. Clearly, this practical appealing property is achieved at the expense of giving up global optimality.

Necessary conditions for the optimal observer are given for the general case where the intensity of the white noise component of the measurement noise may be singular. The solution consists of a modified Riccati and a Lyapunov equation coupled by two projection matrices. The optimal gain is then given explicitly in terms of the nonnegative definite matrices which are the solutions of those equations. In the nonsingular case, one projection, which is due to the singularity of the problem, vanishes and the equations are coupled by only one projection.

Manuscript received September 10, 1989. Paper recommended by Past Associate Editor, M. Gevers.

The author is with the Department of Mechanical Engineering, Technion-Israel Institute of Technology, Haifa 32000, Israel.

IEEE Log Number 9036547 